\title{
Flavored extended instanton in QCD
}

\section{A. Gorsky ${ }^{a, b}$ and A. Grekov ${ }^{c, d}$}

${ }^{a}$ Institute for Information Transmission Problems of the Russian Academy of Sciences, B. Karetnyi 19, Moscow, Russia

${ }^{b}$ Moscow Institute of Physics and Technology, Institutskyi per 9, Dolgoprudny 141700, Russia

${ }^{c}$ Physics Department, Stony Brook University, Stony Brook, NY, U.S.A.

${ }^{d}$ Steklov Mathematical Institute of Russian Academy of Sciences, Gubkina str. 8, Moscow 119991, Russia

E-mail: gorsky@itep.ru, grekovandrew@mail.ru

ABSTRACT: We discuss new flavored space-like defects in confined QCD which can be considered as the Euclidean extended instantons carrying the topologically quantized currents. We focus on the simplest 1d space-like defect - the S-Skyrmion solution extended in one space coordinate and localized in Euclidean time. It can be identified both in the holographic QCD and in the Chiral Perturbation Theory(ChPT). The Skyrmion charges get transformed into the corresponding currents for S-Skyrmion. The analogy with the Thouless pump and the quantum phase slip phenomena is mentioned.

KEywords: Chiral Lagrangians, Confinement, Gauge-gravity correspondence

ARXIV EPRINT: 1910.06304 


\section{Contents}

1 Introduction 1

2 Skyrmion as flavor instanton 3

3 S-Skyrmion in holographic QCD and ChPT 4

3.1 Currents 4

$\begin{array}{lll}3.2 & \text { S-Skyrmion from the dyonic instanton } & 6\end{array}$

3.3 Finite worldline of S-particle $\quad 7$

3.4 Finite temperature 8

3.5 Extended instanton and lattice QCD 9

$\begin{array}{lll}3.62 \mathrm{~d} \text { analogue } & 10\end{array}$

$\begin{array}{lll}3.7 & \text { Analogy with the Thouless pump phenomenon } & 10\end{array}$

4 Multiple space-like defects $\quad 12$

$\begin{array}{ll}4.1 \text { Towards the monopole string } & 12\end{array}$

$\begin{array}{ll}4.2 & \text { Time crystal and S-branes } \\ \end{array}$

5 Conclusion 13

\section{Introduction}

The space-like branes or S-branes have been suggested in [1] in field theory and in the string theory framework. These objects were identified as defects of different codimensions localized in time. The conventional instanton localized in the Euclidean time is the simplest example. The interpretation of the extended space-like defects is a subtle issue and two scenarios have been suggested in [2]. Both of them involves the brane configurations with tachyons - unstable D-brane or $\bar{D} p-D p$ state. The potential for tachyon field supports the kink-like solution, and the space-like brane is interpreted as a tachyon kink in the time direction. It describes some process in field or string theory, in particular the decay of unstable branes into strings [3-5]. The precise example of a creation of the time-like objects from the S-branes has been found in [6].

We shall focus on the similar objects in the conventional QCD in confined phase where baryon is identified as the Skyrmion [7]. In the ChPT it is solution to the equation of motion with a baryonic charge which provides its topological stability. Upon the proper identification of the electromagnetic current $[8]$ it can be shown that it can carry an electric charge as well and enjoys the fermionic statistics [9] due to the 5d Chern-Simons term for the flavor group. The stabilization of a size is provided by the Skyrme term. The solution can be obtained from the holonomy of the instanton solution if one additional artificial 
dimension is added $[10,11]$. This old observation looked a bit puzzling for a while but it gets the clear-cut explanation in the 5d holographic QCD [12]. Baryon aka Skyrmion is nothing but the instanton solution in the holographic QCD on the worldvolume of flavor $N_{f}$ D8 branes [13] which is localized in three space coordinates and radial holographic coordinate $r$. Hence it is extended in time direction and can be considered as a particle. The instanton charge gets identified with the baryonic charge. If the chiral condensate is taken into account for the Skyrmion solution the dyonic instanton in the holographic QCD should be considered [14, 15]. The dyonic instanton solution involves the nontrivial space profile for the tachyonic scalar in the bi-fundamental representation. Baryonic mass becomes the chiral condensate dependent $[14,15]$ and a partial restoration of the chiral symmetry in a core of the solution takes place.

More recently the different picture for the high-spin baryon was suggested [16]. The baryon for $N_{f}=1$ can be thought of as the finite $\eta^{\prime}$ domain wall with the chiral boundary excitations very much as the quantum Hall droplet. A baryon with high spin was identified as the chiral excitation at the boundary and a baryon charge was related to the 2 -form symmetry current.

In this paper we consider new topologically nontrivial solutions in 5d holographic QCD which are flavor instantons localized in Euclidean time therefore being the examples of space-like defects or "extended instantons". Our main example will be the space-like S-Skyrmion that is the defect with one-dimensional worldsheet extended for example in $x_{3}$ coordinate. Its topological charge describes the map of the asymptotics of $\left(r, t_{E}, x_{1}, x_{2}\right)$ 4-dimensional space into the flavor gauge group. Contrary to the Skyrmion when the topological charge yields the baryonic charge density the space component of the quantized topological current is generated. Hence we obtain a kind of instant one-dimensional defect carrying the topological quantized current in the conventional confined QCD. We question if S-Skyrmion enjoys the counterparts of the phenomena familiar for the Skyrmion: electric and axial charges, fermionic statistics, induced decay in the monopole background. We shall argue that a S-Skyrmion hosts the electric and axial currents. The fermionic statistics of the Skyrmions gets mapped into the specific properties of the S-Skyrmion as well. The possible analogue of the Callan-Rubakov effect is suggested.

The topologically quantized currents we consider have some analogy with the Thouless pump phenomenon [17] which concerns the topologically quantized current at the interval. The quantization is supported by the peculiar topological invariant which involves the integration over the period of the external drive. Physically if there is periodic drive applied to the insulator of the finite size the charges of the opposite sign at the boundaries are generated. Recently the related effect, when the topologically quantized work occurs, has been found $[18,19]$. In this case the quantized work of the opposite sign has been performed at the boundaries of the system with the gapped bulk. One more example of the similar nature is the quantum phase slip phenomenon in $1+1$ dimensions [20].

In lattice QCD these configurations should provide the finite contribution into the partition function since the periodic boundary conditions are imposed at the Euclidean space coordinates. In fact the non-perturbative $1 \mathrm{~d}$ defects with the peculiar properties have been found in lattice QCD long time ago (see [21] for the review). They have been 
named as percolating monopoles however it is unclear if these configurations are related to the solutions which we shall describe in this paper.

The paper is organized as follows. First we remind the realization of Skyrmion as the instanton in holographic QCD. In section 3 we consider the S-Skyrmion in holographic QCD and in ChPT and discuss its properties. Section 4 is devoted to the interpretation of array of S-Skyrmions while open questions are formulated in Conclusion.

\section{Skyrmion as flavor instanton}

Let us recall the instanton realization of the baryon in the holographic QCD. In the WittenSakai-Sugimoto(WSS) [22-24] model at $T=0$ the holographic background looks as the cigar-like geometry involving coordinates $(r, \phi)$ supplemented with sphere $S^{4}$ and fourdimensional Minkowski space-time. The flavor degrees of freedom are introduced by adding $N_{f} D 8-\bar{D} 8$ branes extended along all coordinates but $\phi$. The theory on the flavor D8 branes upon the dimensional reduction on $S^{4}$ yields the 5-dimensional Yang-Mills theory with $\mathrm{SU}\left(N_{f}\right)_{R} \times \mathrm{SU}\left(N_{f}\right)_{L}$ gauge group supplemented with the Chern-Simons term. The action reads as

$$
S=\sigma \int d^{4} x d z\left(h(z) \operatorname{Tr} F_{\mu \nu}^{2}+g(z) \operatorname{Tr} F_{\mu z}^{2}\right)+S_{C S}
$$

where $\mu, \nu=1,2,3,4$, the metric factors are

$$
h(z)=\left(1+z^{2}\right)^{1 / 3} \quad g(z)=\left(1+z^{2}\right)
$$

and $\sigma$ is expressed through the t'Hooft coupling $\lambda$ as $\sigma=a \lambda N_{c}, a=\frac{1}{216 \pi^{3}}$.

The baryon in the WSS model is identified as the D4 brane wrapped around $S^{4}$ and extended in the time direction. In terms of the 5d YM theory with the flavor gauge group the baryon is the instanton solution localized in $\left(z, x_{1}, x_{2}, x_{3}\right)$ coordinates. Consider for example $N_{f}=2$ case and separate the $\mathrm{U}(2)$ flavor gauge field on $\mathrm{D} 8$ branes into the $\mathrm{SU}(2)$ field $A(x, z)$ and $\mathrm{U}(1)$ field $B(x, z)$. The solution for the instanton sitting around $(x=0, z=0)$ reads as

$$
A_{\mu}=-i f(\eta) g_{\mathrm{inst}}(x, z) \partial_{\mu} g_{\mathrm{inst}} \quad A_{0}(x, z)=0 \quad f(\eta)=\frac{\rho^{2}}{\eta^{2}+\rho^{2}}
$$

where

$$
\begin{aligned}
g_{\text {inst }} & =\frac{z-i \vec{x} \vec{\tau}}{\sqrt{(z)^{2}+|\vec{x}|^{2}}} & & \\
B_{i}(x, z) & =0 & B_{0}(x, z) & =-\frac{1}{8 \pi^{2} a \lambda \eta^{2}}\left[1-\frac{\rho^{4}}{\left(\eta^{2}+\rho^{2}\right)^{2}}\right]
\end{aligned}
$$

where $\eta^{2}=z^{2}+|\vec{x}|^{2}$ and $\rho$ is the instanton size. This solution is nothing but the Skyrmion solution and it realizes old Atiyah-Manton interpretation [10, 11]. The BPST instanton can be used as a good approximation since it was argued in [25-28] that the solution is mainly localized around $z=0$ where the wrap factor can be neglected. The the mass and radius of the instanton solution in $(x, z)$ space is

$$
M=8 \pi^{2} \sigma+\sqrt{\frac{2}{15}} N_{c} \quad \rho_{\text {inst }}=\frac{1}{8 \pi^{2} a \lambda} \sqrt{6 / 5}
$$


The $5 \mathrm{~d}$ CS term provides its fermionic statistics [8]. The baryonic charge B gets identified as

$$
B=\int d^{3} x d r\left(\operatorname{Tr} F_{L} \tilde{F}_{L}-\operatorname{Tr} F_{R} \tilde{F}_{R}\right)
$$

where $r$ is proportional to $z$.

The holographic model yields the Chiral Lagrangian in the conventional low-energy QCD and reasonable values of the low-energy parameters [23, 24]. To this aim it is necessary to fix the boundary conditions for all components of the flavor gauge field

$$
A_{M}(x, z) \rightarrow 0(z \rightarrow \pm \infty) \quad M=(0,1,2,3,4, z)
$$

and introduce the holonomy along the radial coordinate

$$
U(x)=P \exp \left(-i \int_{-\infty}^{+\infty} d z A_{z}(x, z)\right)
$$

It is field $U(x)$ gets identified with a matrix of Goldstone mesons in the Chiral Lagrangian. The rest of the flavor gauge fields in the holographic model amount to the massive mesons in low-energy QCD.

The Skyrmion solution can be derived in the conventional ChPT with Lagrangian

$$
L=-\frac{F_{\pi}^{2}}{16} \operatorname{Tr}\left(U \partial_{\nu} U^{\dagger}\right)^{2}+\frac{1}{32 c^{2}} \operatorname{Tr}\left(\left[U \partial_{\mu} U^{\dagger}, U \partial_{\nu} U^{\dagger}\right]\left[U \partial_{\mu} U^{\dagger} U \partial_{\nu} U^{\dagger}\right]\right)
$$

where the second Skyrme term provides the stabilization of the Skyrmion. The baryonic charge in ChPT is expressed in terms of the unitary matrix field $U(x, t)$ of the Goldstone pions as

$$
B=\int d^{3} x \operatorname{Tr}\left(U^{-1} d U\right)^{3} \quad U(x, t)=\exp \left(\frac{i \pi^{a} t^{a}}{F_{\pi}}\right)
$$

Its topological nature is supported by the nontrivial $\pi_{3}(\mathrm{SU}(2))=Z$ since asymptotic condition $U\left(x_{1}, x_{2}, x_{3} \rightarrow \infty\right) \rightarrow 1$ provides the mapping of $S_{3}$ into the diagonal flavor group. The solution has the electric charge due to $N_{c}$ fundamental strings attached to the baryonic vertex or D4 brane [29]. The mass of the Skyrmion is $M \propto \frac{F_{\pi}}{c}$ while its radius is $r_{s}=\frac{1}{F_{\pi} c}$. The Skyrmion enjoys the axial and tensor charges as well.

It is possible also to include the chiral symmetry breaking condensate into the $5 \mathrm{~d}$ action explicitly via the boundary behavior of the additional tachyonic scalar field $\mathrm{X}$ in the bi-fundamental representation. In this case the baryon becomes the dyonic instanton solution with two quantum numbers and the tachyon field has nontrivial kink-like profile in the space. The mass of the dyonic instanton in some regime is determined by the chiral condensate [14, 15]. One could say that in the dyonic instanton representation of the Skyrmion the chiral symmetry breaking is partially restored at its core.

\section{$3 \quad$ S-Skyrmion in holographic QCD and ChPT}

\subsection{Currents}

Turn now to the space-like S-Skyrmion solution in QCD. First, perform the Wick rotation and consider $R^{4}$ instead of the Minkowski space. The D4 brane representing the S-Skyrmion 
is wrapped around $S^{4}$ and extended in $x_{3}$ coordinate. Since D4 brane shares all coordinates with D8 branes it amounts to the instanton-like solution in the flavor gauge theory as for any $D p-D(p+4)$ system. However this instanton has the different interpretation in comparison with the baryon since it is localized in the Euclidean time and is extended in one space dimension.

Consider the BPST solution in the 5d flavor YM theory localized in $\left(t_{E}, r, x_{1}, x_{2}\right)$. It looks the same as the standard Skyrmion-instanton however is extended along say $x_{3}$ coordinate instead of time coordinate.

$$
A=A_{\text {inst }}\left(t_{E}, x_{1}, x_{2}, z\right) \quad B=B_{\text {inst }}\left(t_{E}, x_{1}, x_{2}, z\right)
$$

The potential for the size of extended instanton is the same as before. Therefore similar to the conventional Skyrmion using the approximate rotational symmetry of the solution we can estimate the energy density of the S-Skyrmion as the mass of the conventional Skyrmion $T=M_{S k}$ and its size in the Euclidean time direction is identified with the radius of the Skyrmion $\delta t_{E}=\rho_{S k}$.

There is topologically conserved current in $5 \mathrm{D}$

$$
J^{5 d}=* \operatorname{Tr} F_{A} \wedge F_{A}, \quad F_{A}=F_{L}-F_{R}
$$

which yields the baryonic charge for the Skyrmion. S-Skyrmion carries non-vanishing current component $J_{3}$, say along $x_{3}$ space coordinate along which it is extended

$$
J_{3}=\int d x_{1} d x_{2} d r d t_{E} \operatorname{Tr} F \tilde{F}=\int_{x_{3}=\text { const }} \operatorname{Tr} F_{A} \wedge F_{A}
$$

In the ChPT the extended instanton solution saturates the topological charge representing $\pi_{3}(\mathrm{SU}(2))$. Upon imposing a asymptotic behavior in the Euclidean space-time $U\left(x_{1}, x_{2}, t_{E} \rightarrow \infty\right) \rightarrow 1$ it maps the three-dimensional sphere into the flavor group. The topological current density substituting the baryonic charge in this case reads as

$$
\tilde{J}_{3}=\int d t_{E} d^{2} x \epsilon_{t 12} \operatorname{Tr}\left(U^{-1} d_{t_{E}} U\right)\left(U^{-1} d_{x_{1}} U\right)\left(U^{-1} d_{x_{2}} U\right)
$$

and involves the integration over Euclidean time and two space-like coordinates $x_{1}, x_{2}$. This means that solution has nontrivial topological current density only for the time-dependent pion fields.

The next question concerns the electric charge of the solution. The following term in the ChPT is relevant for the electromagnetic current evaluated at our solution

$$
L_{w z w}=-\frac{N_{c}}{32 \pi^{2}} \epsilon^{\mu \nu \alpha \beta} \operatorname{Tr} Q\left(U^{-1} \partial_{\mu} U U^{-1} \partial_{\alpha} U U^{-1} \partial_{\beta} U\right) A_{\nu}
$$

We can extract this contribution also from 5 d action involving the $\mathrm{U}(1)$ connection as well and get the term in the $4 \mathrm{~d}$ action

$$
S_{\mathrm{int}}=N_{c} \int d x_{3} A_{3}^{\mathrm{U}(1)} J_{3}
$$


which means that the S-Skyrmion is coupled to the abelian gauge potential. The $N_{c}$ factor in the standard Skyrmion tells that the $N_{c}$ fundamental strings are attached to the baryonic vertex supporting its composite nature as state build from $N_{c}$ quarks with fractional baryonic charge. Similarly we could assume that S-Skyrmion has the composite nature and can be thought as the baryonic vertex with $N_{c}$ fundamental strings supporting the fractional topologically quantized current. The $N_{c}$ fundamental strings attached to vertex have $\left(x_{3}, r\right)$ worldsheet coordinates.

It is useful to compare this current with the electric current in the external magnetic field via chiral magnetic effect(CME) [30]. In the hadronic phase the CME induced electric current has been discussed in [31]. It needs for the chiral disbalance induced by the chiral chemical potential or the time dependent pseudoscalar field and external magnetic field. In our study we have electric current induced by the time-dependent pion classical configuration in the Euclidean space without external magnetic field. Remind that the solution is topologically non-trivial due to $\pi_{3}(\mathrm{SU}(2))=\mathbb{Z}$, hence we can integrate the current density and obtain non-vanishing $J_{3}$ current during the whole process. It is proportional to the topological invariant therefore is quantized and do not vanish for our extended instanton solution.

The Skyrmion carries the axial charge which can be easily holographycally seen as follows. If we treat the radial coordinate as time the canonical momentum for the axial gauge field gets modified and reads as

$$
\Pi_{\mu}^{A}=E_{\mu}^{A}+N_{c} K_{\mu}^{A}
$$

where the second term follows from the CS term. Now consider the Gauss law constraint in the axial channel $\partial_{\mu} E_{\mu}^{A}=0$, express the electric field in terms of the canonical momentum and impose this constraint on the Skyrmion state

$$
\left.\left(\partial_{\mu} \frac{\delta}{\delta A_{\mu}}-N_{c} F \wedge F\right) \mid \text { Skyrmion }\right\rangle=0
$$

The variation over the axial gauge field yields the axial current at the boundary, hence the Skyrmion which has the non-vanishing value of the baryonic charge has the axial charge as well. In fact this is the analogue of the Witten effect when the monopole acquires the electric charge in the presence of the $\theta$-term due to the modification of the canonical momentum.

We can apply the same logic for the S-Skyrmion which carries the topological current. The only difference is that we integrate the Gauss law constraint over $\left(x_{1}, x_{2}, t_{E}\right)$. Hence S-Skyrmion carries the axial current as well.

\subsection{S-Skyrmion from the dyonic instanton}

One can consider more general solution for the description of the S-Skyrmion from the $5 \mathrm{~d}$ viewpoint. In the conventional Skyrmion case the dyonic instanton solution has been numerically found in $[14,15]$ and takes into account the chiral condensate via the boundary condition for the bi-fundamental tachyonic scalar $\mathrm{X}$ at $z \rightarrow 0$

$$
X=\frac{1}{2}\left(m z+\chi z^{3}+\ldots\right) \quad\langle\bar{q} q\rangle=\frac{N_{c}}{2 \pi} \chi
$$


where $m$ is the quark mass. The bifundamental tachyonic scalar emerges from the mode of the string connecting flavor $\bar{D} 8-D 8$ branes. The Skyrmion solution in this case involves the nontrivial profile of the tachyon scalar field in the space and it was argued that the chiral condensate tends to vanish in a Skyrmion core.

We could consider the similar dyonic instanton solution for the S-Skyrmion. The situation starts to remind the initial interpretation of the generic S-brane as the tachyon kink in the time direction in the $\bar{D} p-D p$ system [1]. The S-brane is assumed to be located at the extremum of the tachyon potential $\mathrm{V}(\mathrm{T})$ at $T=0$. The tachyon field involved into the dyonic instanton solution has the kink profile in the Euclidean time direction and S-Skyrmion is located around $X=0$ where the chiral condensate tends to vanish. The energy density of the S-Skyrmion will depend on the value of the chiral condensate like in $[14,15]$ for the conventional Skyrmion.

The situation resembles the quantum phase slip phenomenon in $1+1$ dimensions. It is to some extend dual to the superconducting current. There is the instantaneous topological current of vortices across the superconducting wire. Microscopically the amplitude of the Cooper condensate vanishes locally and the phase of the condensate rapidly rotates at $2 \pi k$. This flip blocks the superconducting current. The total vorticity does not change but the vortices of the opposite topological charges emerge at two boundaries.

In our situation we have the tunneling topological current "across the sample" as well. Let us assume the S-Skyrmion is extended in the finite dimension. In the dyonic instanton realization of the S-Skyrmion the analogy is very close - we have the small region at the core of the dyonic instanton with the restoration of the chiral symmetry which supports a tunneling of the topological charge. In our case the topological charge is the baryonic charge hence instead of the emerging vorticity at boundaries in $1+1$ we expect the opposite baryonic charges at the boundary of the interval. In our case the pions play the role of the phases of the chiral condensate and the topological invariant measures the jump of the pionic phase as well. However the analogy with the phase slip phenomena deserves further study.

The conventional Skyrmion is fermion due to the 5d CS term. Hence it is natural to address the question concerning the counterpart of the fermionic statistics for S-Skyrmion. Instead of rotation in $\left(x_{1}, x_{2}, x_{3}\right)$ we have similar topological arguments concerning rotation in $\left(t_{E}, x_{2}, x_{3}\right)$ in Euclidean space. Naively one could imagine that a kind of Pauli principle forbids several S-Skyrmions at one point in $\left(t_{E}, x_{2}, x_{3}\right)$ space. However there are some subtleties concerning the analytic continuation into the Euclidean space hence we postpone this question for the separate study.

\subsection{Finite worldline of S-particle}

The action of the S-particle solution extended in $x_{3}$ is proportional to the length of $x_{3}$ coordinate - so for non-compact $x_{3}$ it is infinite, hence to have the finite action we need to consider the S-particle with the finite length. This can be achieved by periodic $x_{3}$ or by imposing the proper boundary condition providing its termination at the ends of finite interval. Consider first the periodic coordinate. Since the space-like particle could be considered as a baryon with $x_{3}$ playing the role of Euclidean time, to find all the possible classical string configurations we need to classify the classical periodic solutions of equations 
of motion for the particle moving in the inverted potential (2.5). It is easy to see that the periodicity requirement leaves only solutions, for which the effective instanton radius $\rho$ does not depend on $x_{3}$ - the particle sitting at the bottom of the potential. However these solutions could still move in the physical space and in the sphere $S^{3}$. The action on such solutions is equal to

$$
S_{\text {string }}=S_{\text {kin }}+L_{x_{3}} \frac{4 \pi}{g_{5 d}^{2}}
$$

where $L_{x_{3}}$ is a size of $x_{3}$ circle.

To discuss the second possibility and explain the possible termination of the SSkyrmion at some value of space coordinate consider the analogous process - the decay of the Skyrmion in time. The key point is that the baryonic current gets modified in the external electromagnetic field and reads as

$$
\begin{aligned}
B_{\nu}= & \frac{1}{24 \pi^{2}} \epsilon^{\mu \nu \alpha \beta} \operatorname{Tr}\left(U^{-1} \partial_{\mu} U U^{-1} \partial_{\alpha} U U^{-1} \partial_{\beta} U\right) \\
& -\frac{1}{8 \pi^{2}} \epsilon^{\mu \nu \alpha \beta} \partial_{\mu}\left[\left(A_{\alpha} \operatorname{Tr}\left(Q\left(U^{-1} \partial_{\beta} U+\partial_{\beta} U U^{-1}\right)\right)\right]\right.
\end{aligned}
$$

In the monopole background the Bianchi identity for the gauge field is violated $\partial_{\nu} \tilde{F}_{\nu \mu}=$ $J_{\mu}^{\text {mon }}$ and the density of the baryon current is not conserved

$$
\partial_{\nu} B_{\nu} \neq 0
$$

Integrating baryon charge over the 3-dimensional space one gets the rate of the baryon decay in the monopole background

$$
\frac{d B}{d t} \propto \rho^{\text {mon }} \partial_{t} \pi^{0}
$$

It is the Skyrmion realization of the Callan-Rubakov effect [32-35]. The unwinding of the Skyrmion occurs through the time dependent pion field. The process has been identified in holography as well [36] where the wrapped D4 Skyrmion gets dissolved in the D6 monopole string.

The S-Skyrmion also undergoes the termination at the S-monopole. To identify this effect assume that the monopole current is directed along $x_{3}$ coordinate hence $J_{3}^{\text {mon }} \neq 0$. The non-conservation of the component of the baryonic current now reads as

$$
\frac{d B_{3}}{d x_{3}}=J_{3}^{\text {mon }} \partial_{x_{3}} \pi^{0}
$$

Integrating this equation over $\int d x_{1} d x_{2} d t_{E}$ we obtain the rate of "termination" of the instanton extended along $x_{3}$ coordinate. Since the S-Skyrmion is represented by the same D4 vertex with the rotated worldsheet the termination of S-brane can occur similarly via dissociation at the D6 brane represented by the monopole string.

\subsection{Finite temperature}

Let us consider the case of finite temperature that is periodic Euclidean time. Since the Euclidean time is involved in our S-Skyrmion, solution gets modified into the caloron. 
The caloron configuration [37-40] for $\mathrm{SU}(N)$ gauge group can be thought as the composite object involving $N$ constituents with magnetic and fractional instanton $1 / N$ charges. The total magnetic charge vanishes while the total instanton charge equals one. The constituents are distributed along the thermal direction at distances dictated by the value of the Polyakov loop.

In the simplest case the caloron solution reads as

$$
A_{\mu}^{a}=\bar{\eta}_{\mu \nu}^{a} \Pi(x) \partial_{\nu} \Pi^{-1}(x)
$$

where $\bar{\eta}_{\mu \nu}^{a}$ - anti-t'Hooft symbol and $\Pi(x)$ has the form:

$$
\Pi(x)=1+\frac{\pi \rho^{2} T}{r} \frac{\sinh (2 \pi r T)}{\cosh (2 \pi r T)-\cos \left(2 \pi t_{E} T\right)}
$$

where $T$ - is temperature, $\rho$ - is a size of a solution and $r^{2}=x_{1}^{2}+x_{2}^{2}+z^{2}$.

In our case we have such caloron solution in the flavor gauge group hence our SSkyrmion gets defragmented into the $N_{f}$ constituents with additional "flavor magnetic" charges and fractional topological numbers. Since the topological number now measures the current this means that the total current can be represented via the finite number of components with the fractional current. Since the S-brane here is the baryonic vertex with $N_{c}$ strings the fractionalization implies the formation of $N_{f}$ groups with $\frac{N_{c}}{N_{f}}$ strings in each group.

\subsection{Extended instanton and lattice QCD}

The lattice QCD deals with the Euclidean space-time with periodic boundary conditions. This set-up is suitable for the search of the S-Skyrmion with quantized currents. Indeed our S-Skyrmion is localized at $T^{3} \times R^{1}$ and the Chern number can be defined in this case. The instanton solution in this geometry gets fractionalized and can be treated as the bound state of the fractional instantons with fractional topological currents. We expect that such $1 \mathrm{~d}$ closed loops extended along one space coordinate should be observed in lattice QCD studies.

In fact the whole Zoo of defects has been observed on the lattice (see [21] for the review). They involve defects with $1 \mathrm{~d}, 2 \mathrm{~d}$ and $3 \mathrm{~d}$ worldvolumes. The $1 \mathrm{~d}$ defects found in the lattice QCD were interpreted as the monopoles, moreover two types of monopole configurations - one of IR nature while the second of UV nature were observed. It would be interesting to compare properties of these observed 1d defects with properties of S-Skyrmion. Note that the holographic classification of QCD defects can be found in [41].

One more point worth to be mentioned. It is known from the lattice QCD that all eigenfunctions of the $4 \mathrm{~d}$ Euclidean Dirac operator are delocalized [42] in confined phase while there is the mobility edge in the deconfined phase (see [43] for the recent holographic interpretation). The low-energy QCD is treated as the random chiral matter and the pion decay constant $F_{\pi}$ defines the diffusion coefficient. We could speculate that the extended instantons of different codimensions could provide the ungapped channels for the delocalization via a kind of percolation mechanism however this point certainly deserves the further study. If it is true it would be a kind of fracton picture (see [44, 45] for review) for the transport of Dirac operator modes. 


\section{$3.62 d$ analogue}

Let us comment on the similar baby S-Skyrmion solution in the $2 \mathrm{~d} N_{f}=1$ QCD within $3 \mathrm{~d} \mathrm{U}(1)_{L} \times \mathrm{U}(1)_{R}$ flavor gauge group in the holographic description [46] involving $(t, x, r)$ coordinates. The Lagrangian of the model involves the tachyonic bi-fundamental scalar similar to the $4 \mathrm{~d}$ case. The conventional vortex solution to the equation of motion in the gauge theory in $(r, x)$ space yields the analogue of the Skyrmion in $2 \mathrm{~d}$ propagating in time and having the topological charge $B_{2}=\int d x A_{x}$ well defined if $x \in S_{1}$.

Now let us make the Wick rotation and consider the vortex solution in the $\left(r, t_{E}\right)$ plane instead. It is localized in Euclidean time and extended in the $x$ coordinate that it is a kind of extended instanton. The topological charge of such space-like defect solution in $3 \mathrm{~d}$ flavor YM gauge theory supplemented with $3 \mathrm{~d}$ CS term reads as

$$
Q_{2}=\int d t_{E} d r * F=\delta \int d t_{E} A_{0}-\delta \int d r A_{r}
$$

where we take into account that the integrand is the total derivative.

What is the interpretation of this solution if any? First note that there is topologically conserved axial 3d current density in the theory

$$
J^{3 d}=*\left(F_{L}-F_{R}\right)
$$

which amounts to the non-vanishing axial current component

$$
J_{x}=Q_{2}
$$

for our solution. Hence we have a impulse-like axial current along the space coordinate.

In the gauge $A_{0}=0$ the phase of the chiral condensate is identified with the $\int d r A_{r}$ hence our topological invariant for the baby $1+1$ S-Skyrmion is just the jump of the phase at the instantaneous topological current. The situation is very similar to the quantum phase slip phenomenon in the superconducting wire. The only difference is that we have substituted the Cooper condensate by the excitonic condensate. In some sense this subsection provides the holographic realization of the quantum phase flip phenomena.

\subsection{Analogy with the Thouless pump phenomenon}

An interesting analogy with the Thouless pump phenomenon [17] worth to be mentioned. It was argued in [17] that for the periodic driving process a pump of a charge at the boundaries of the gapped space interval can be identified and is topologically protected. It is to some extend the non-stationary analogue of the TKNN invariant defined for the stationary case which yields the Hall conductivity. The invariant expression for the current reads as $[17]$

$$
Q=\frac{1}{T} \int_{0}^{T} d t d^{2} k T r\left(U^{-1} \partial_{t} U U^{-1} \partial_{k_{1}} U U^{-1} \partial_{k_{2}} U\right)
$$

and it involves the nontrivial mapping of the $\left(t, k_{1}, k_{2}\right)$ space into the group of rotation of the ground state of the system. It is a version of the Chern number for the Berry connection. The corresponding current is quantized due to its topological nature. 
More recently the similar Thouless pump phenomenon has been found for the topologically quantized work instead of the current flow [18]. Moreover the Chern number can be identified not only necessarily for the momentum space but for the coordinate space as well [19]. The corresponding expression for the Chern number yielding the topologically quantized work reads as

$$
Q=\frac{1}{T} \int_{0}^{T} d t d^{2} x \operatorname{Tr}\left(U^{-1} \partial_{t} U U^{-1} \partial_{x_{1}} U U^{-1} \partial_{x_{2}} U\right)
$$

The total energy is conserved of course but there is topologically quantized work done at the edges with the opposite signs.

Our expression for the topologically quantized current (3.3) is of the same nature. The only difference is that we find the extended instanton not in Minkowski but in the Euclidean space hence its interpretation is a bit different. However as we have mentioned above the tunneling interpretation of the instantaneous current is relevant for the quantum phase slip.

In the Thouless pump or energy pump the Berry phase interpretation involves the matrix of unitary rotation of the state $U(p, t)$ or $U(x, t)$. In the Thouless pump case the wave function gets adiabatically rotated under the periodic driving force and depends on the $2 \mathrm{~d}$ momentum space

$$
\Psi(p, t+T) \rightarrow U(p, t, T) \Psi(p, t)
$$

or via similar rotation by $U(x, t, T)$ in the energy pump case. The $\left(t, p_{1}, p_{2}\right)$ or $\left(t, x_{1}, x_{2}\right)$ are considered as the parameter space in the Berry phase interpretation and the corresponding Chern number for the Berry connection in this space plays the role. One can also consider the external driving force instead of time.

We could question if the Berry phase interpretation of our invariant is possible. The matrix $U(x, t)$ involved into the Chern number of the S-Skyrmion indeed can be interpreted as the chiral rotation of the ground state in the chirally broken phase. Namely the Chiral Lagrangian can be derived from the quark fermionic determinant if we assume that the matrix of Goldstone mesons $U$ provide the chiral phase of the quark mass [47]. The corresponding covariant derivative in quark Lagrangian reads as

$$
D_{\mu}(A, U)=i \partial_{\mu}+A_{\mu} \otimes I+I \otimes U \partial_{\mu} U^{\dagger}
$$

where A is a gluon field, the first factor refers to color space and the second to the flavor space. Therefore from the quark viewpoint a kind of Berry connection can be defined for the external time-varying pion field and our invariant can be considered as its Chern number. Note that in our case contrary to the energy pump phenomena a period in Euclidean time is infinite. 


\section{Multiple space-like defects}

\subsection{Towards the monopole string}

As it was discussed in [48] the BPS monopole solution could be obtained as an infinite sequence of instantons:

$$
\Pi\left(x, t_{E}\right)=\sum_{n=-\infty}^{+\infty} \frac{1}{\beta^{2}\left(r^{2}+\left(t_{E}-\frac{2 \pi n}{\beta}\right)^{2}\right)}
$$

after a special gauge transformation with the help of the group element:

$$
U\left(x, t_{E}\right)=\exp \left(-i \tau_{a} \frac{x_{a}}{|x|} \theta\right)
$$

which makes the solution

$$
A_{\mu}^{a}=\bar{\eta}_{\mu \nu}^{a} \Pi(x) \partial_{\nu} \Pi^{-1}(x)
$$

time-independent, where

$$
\theta=\arctan \frac{\sin \left(\beta t_{E}\right) \sinh (\beta r)}{\cosh (\beta r) \cos \left(\beta t_{E}\right)-1}
$$

We could try to perform the analogous procedure in our case for the S-Skyrmion constant in $x_{3}$. The only problem is the change of the asymptotic behavior of our solution as $z \rightarrow \infty$ under the gauge transform (4.2). We need to check that it is compatible with the approximation of the flat $\mathrm{z}$ coordinate near $z=0$, made in $[25,26]$. The form of the gauge transformation (4.2) will be valid only for small values of $z$. We shall investigate this subtle point elsewhere. The energy density of such "monopole string" is finite:

$$
\frac{d E}{d x_{3}}=\frac{d S}{d t_{E} d x_{3}}=\frac{4 \pi \beta}{g_{5 d}^{2}}
$$

\subsection{Time crystal and S-branes}

Recently the idea of the "time crystal" that is the dynamically organized time periodicity has been forwarded $[49,50]$ (see [51] for review). It was recognized that the time crystal is impossible in the equilibrium state however the possibility for such state at non-equilibrium can not be excluded. Moreover the scenario for the time crystal in the many body localized state with periodic quench has been suggested and observed experimentally. Such system develops periodicity in time with period different from the period of the external drive. Let us remark that the S-branes can serve as the building blocks in a kind of the time crystal in the constant external electric field in the Euclidean space-time. Let us assume for example that the electric field is added to $1+1$ theory with fermions and the Schwinger pair creation is considered. The leading Euclidean bounce is just the circle [52] with negative mode however more general configuration involving the multiple parallel extended instantons can be considered as well. Such configuration has been discussed in [53]. To some extend one could say that the fermion-antifermion pair interact instantly via the extended instanton which can be thought of as the bound state. 
A bit loosely one could say that we have the array of the interacting Wilson loops in the $2 \mathrm{~d}$ Euclidean space-time. Due to the interaction between the Wilson loops we get a kind of neutral S-meson. Similarly we can get in higher dimensions the similar bounce in the external magnetic field describing the monopole pair creation. In this case we get the interacting t'Hooft loops and the magnetic S-meson.

The emerging period in the time direction depends on the ratio of the masses of particles and the tension of the S-meson. Upon summation over the time ladder the emerging period looks like the Unruh temperature for the accelerated particle in the external electric field [53]. We get structure similar to the time crystal in the Euclidean time but there is the remnant of the dynamical period in the Minkowski time upon the analytic continuation of the caterpillar bounce back to the Minkowski time. Indeed the analytic continuation remembers the periodicity in the Euclidean time and to some extend the Unruh temperature is the counterpart of the time crystal period in the Euclidean space. Note that our picture is different from the holographic picture for Floquet states based on the Schwinger process in the time-periodic external electric field which has been suggested in [54].

\section{Conclusion}

In this note we have found a new space-like defect — S-Skyrmion in the confined Euclidean QCD which can be thought of as the flavored extended instanton. The solution is localized in time and hosts the several types of currents which are the counterparts of the Skyrmion charges - baryonic, electric and axial. The baryonic current of the S-Skyrmion is quantized in the proper normalization and is topologically protected. If we use the dyonic instanton solution in holographic QCD for S-Skyrmion the situation is quite close to the initial formulation of the position of S-brane at the extremum of the tachyonic potential.

This topologically quantized current has similarities with the Thouless current pump phenomena for the periodically driven systems since the similar Chern number does the job. In the Thouless case the electric charges emerge at the boundaries of the gapped bulk. One more phenomena of the similar nature - the quantum phase slip involves the "creation" of the vortex pair at the edges of the gapped bulk via tunneling. We could speculate that the S-Skyrmion process could produce the pair of magnetic monopoles at the edges since we have shown that the S-Skyrmion can terminate at the monopole via the dual version of the Callan-Rubakov effect.

Here we have considered the simplest example of $1 \mathrm{~d}$ extended instanton but the similar solutions involving the space-like defects with $2 \mathrm{~d}$ and $3 \mathrm{~d}$ worldvolumes do exist as well. The lattice studies indicate that they exist on the equal footing with $1 \mathrm{~d}$ defects and we hope to discuss them elsewhere. We expect that the properties of the S-Skyrmion can be quite precisely analyzed in lattice QCD. Presumably the space-like defects could also play the role in explanation of the well established counterintuitive delocalization of all Dirac operator modes in confined QCD in a kind of fracton picture.

The role of these solutions at non-vanishing temperature and chemical potential in particular near the deconfinement phase transition should be clarified. It would be also interesting to investigate carefully the structure of the moduli spaces of such class of topological solutions and the possibility of the network involving the defects of different codimensions to 
exist. Since the S-defects generically carry the p-form current it is necessary to investigate carefully the current matching in the generic network. Some work in this direction has been recently done in [55]. The S-brane can produce the conventional time-like defects hence the possibility of more general networks in the Euclidean QCD certainly has to be investigated.

The moduli space of conventional Skyrmion are quantized yielding the spectrum of excitations. Hence we expect that the similar quantization of the S-Skyrmion moduli space has to be performed and will provide the tower of the excited states of S-Skyrmion.

The chiral condensate is the analogue of a exciton condensate in the solid state physics. Hence it would be interesting to elaborate the similar S-Skyrmions in that context. In particular the S-Skyrmions presumably could yield a kind of quantum phse slip phenomenon in the excitonic condensate.

\section{Acknowledgments}

The work of A.Gor. was supported by Basis Foundation fellowship and RFBR grant 19-0200214. The work of A.Gr. was supported by the Russian Science Foundation under grants 19-11-00062 and 18-02-01081. A.Gor. thanks Simons Center for Geometry and Physics at Stony Brook University where the work has been completed for the hospitality and support.

Open Access. This article is distributed under the terms of the Creative Commons Attribution License (CC-BY 4.0), which permits any use, distribution and reproduction in any medium, provided the original author(s) and source are credited.

\section{References}

[1] M. Gutperle and A. Strominger, Space-like branes, JHEP 04 (2002) 018 [hep-th/0202210] [INSPIRE].

[2] A. Sen, Rolling tachyon, JHEP 04 (2002) 048 [hep-th/0203211] [INSPIRE].

[3] A. Strominger, Open string creation by S branes, Conf. Proc. C 0208124 (2002) 20 [hep-th/0209090] [INSPIRE].

[4] D. Gaiotto, N. Itzhaki and L. Rastelli, Closed strings as imaginary D-branes, Nucl. Phys. B 688 (2004) 70 [hep-th/0304192] [INSPIRE].

[5] N.D. Lambert, H. Liu and J.M. Maldacena, Closed strings from decaying D-branes, JHEP 03 (2007) 014 [hep-th/0303139] [INSPIRE].

[6] K. Hashimoto, P.-M. Ho, S. Nagaoka and J.E. Wang, Time evolution via S-branes, Phys. Rev. D 68 (2003) 026007 [hep-th/0303172] [InSPIRE].

[7] E. Witten, Baryons in the 1/N expansion, Nucl. Phys. B 160 (1979) 57 [InSPIRE].

[8] E. Witten, Global aspects of current algebra, Nucl. Phys. B 223 (1983) 422 [INSPIRE].

[9] E. Witten, Current algebra, baryons and quark confinement, Nucl. Phys. B 223 (1983) 433 [INSPIRE].

[10] M.F. Atiyah and N.S. Manton, Skyrmions from instantons, Phys. Lett. B 222 (1989) 438 [INSPIRE].

[11] M. Atiyah and P. Sutcliffe, Skyrmions, instantons, mass and curvature, Phys. Lett. B 605 (2005) 106 [hep-th/0411052] [INSPIRE]. 
[12] M. Eto, M. Nitta, K. Ohashi and D. Tong, Skyrmions from instantons inside domain walls, Phys. Rev. Lett. 95 (2005) 252003 [hep-th/0508130] [INSPIRE].

[13] D.T. Son and M.A. Stephanov, QCD and dimensional deconstruction, Phys. Rev. D 69 (2004) 065020 [hep-ph/0304182] [InSPIRE].

[14] A. Gorsky and A. Krikun, Baryon as dyonic instanton, Phys. Rev. D 86 (2012) 126005 [arXiv: 1206.4515] [INSPIRE].

[15] A. Gorsky, S.B. Gudnason and A. Krikun, Baryon and chiral symmetry breaking in holographic QCD, Phys. Rev. D 91 (2015) 126008 [arXiv:1503.04820] [INSPIRE].

[16] Z. Komargodski, Baryons as quantum Hall droplets, arXiv:1812.09253 [INSPIRE].

[17] D.J. Thouless, Quantization of particle transport, Phys. Rev. B 27 (1983) 6083.

[18] M.H. Kolodrubetz, F. Nathan, S. Gazit, T. Morimoto and J.E. Moore, Topological Floquet-Thouless energy pump, Phys. Rev. Lett. 120 (2018) 150601.

[19] B. Mera, K. Sacha and Y. Omar, Topologically protected quantization of work, Phys. Rev. Lett. 123 (2019) 020601.

[20] A. Bezryadin, C.N. Lau and M. Tinkham, Quantum suppression of superconductivity in ultrathin nanowires, Nature 404 (2000) 971.

[21] V.I. Zakharov, Lower-dimension vacuum defects in lattice Yang-Mills theory, Phys. Atom. Nucl. 68 (2005) 573 [hep-ph/0410034] [InSPIRE].

[22] E. Witten, Anti-de Sitter space, thermal phase transition and confinement in gauge theories, Adv. Theor. Math. Phys. 2 (1998) 505 [hep-th/9803131] [INSPIRE].

[23] T. Sakai and S. Sugimoto, Low energy hadron physics in holographic QCD, Prog. Theor. Phys. 113 (2005) 843 [hep-th/0412141] [InSPIRE].

[24] T. Sakai and S. Sugimoto, More on a holographic dual of QCD, Prog. Theor. Phys. 114 (2005) 1083 [hep-th/0507073] [INSPIRE].

[25] H. Hata, T. Sakai, S. Sugimoto and S. Yamato, Baryons from instantons in holographic QCD, Prog. Theor. Phys. 117 (2007) 1157 [hep-th/0701280] [InSPIRE].

[26] K. Hashimoto, T. Sakai and S. Sugimoto, Holographic baryons: static properties and form factors from gauge/string duality, Prog. Theor. Phys. 120 (2008) 1093 [arXiv: 0806.3122] [INSPIRE].

[27] A. Pomarol and A. Wulzer, Stable skyrmions from extra dimensions, JHEP 03 (2008) 051 [arXiv:0712.3276] [INSPIRE].

[28] A. Pomarol and A. Wulzer, Baryon physics in holographic QCD, Nucl. Phys. B 809 (2009) 347 [arXiv: 0807.0316] [INSPIRE].

[29] E. Witten, Baryons and branes in anti-de Sitter space, JHEP 07 (1998) 006 [hep-th/9805112] [INSPIRE].

[30] K. Fukushima, D.E. Kharzeev and H.J. Warringa, The chiral magnetic effect, Phys. Rev. D 78 (2008) 074033 [arXiv: 0808.3382] [INSPIRE].

[31] K. Fukushima and K. Mameda, Wess-Zumino-Witten action and photons from the chiral magnetic effect, Phys. Rev. D 86 (2012) 071501 [arXiv:1206.3128] [INSPIRE].

[32] V.A. Rubakov, Superheavy magnetic monopoles and proton decay, JETP Lett. 33 (1981) 644 [Pisma Zh. Eksp. Teor. Fiz. 33 (1981) 658] [inSPIRE].

[33] V.A. Rubakov, Adler-Bell-Jackiw anomaly and fermion number breaking in the presence of a magnetic monopole, Nucl. Phys. B 203 (1982) 311 [INSPIRE]. 
[34] C.G. Callan Jr., Monopole catalysis of baryon decay, Nucl. Phys. B 212 (1983) 391 [INSPIRE].

[35] C.G. Callan and E. Witten, Monopole catalysis of skyrmion decay, Nucl. Phys. B 239 (1984) 161.

[36] D.K. Hong, K.-M. Lee, C. Park and H.-U. Yee, Holographic monopole catalysis of baryon decay, JHEP 08 (2008) 018 [arXiv:0804.1326] [INSPIRE].

[37] B.J. Harrington and H.K. Shepard, Periodic Euclidean solutions and the finite temperature Yang-Mills gas, Phys. Rev. D 17 (1978) 2122 [InSPIRE].

[38] B.J. Harrington and H.K. Shepard, Thermodynamics of the Yang-Mills gas, Phys. Rev. D 18 (1978) 2990 [INSPIRE].

[39] D.J. Gross, R.D. Pisarski and L.G. Yaffe, QCD and instantons at finite temperature, Rev. Mod. Phys. 53 (1981) 43 [inSPIRE].

[40] T.C. Kraan and P. van Baal, Periodic instantons with nontrivial holonomy, Nucl. Phys. B 533 (1998) 627 [hep-th/9805168] [INSPIRE].

[41] A.S. Gorsky, V.I. Zakharov and A.R. Zhitnitsky, On classification of QCD defects via holography, Phys. Rev. D 79 (2009) 106003 [arXiv:0902.1842] [INSPIRE].

[42] A.M. Garcia-Garcia and J.C. Osborn, Chiral phase transition in lattice QCD as a metal-insulator transition, Phys. Rev. D 75 (2007) 034503 [hep-lat/0611019] [INSPIRE].

[43] A. Gorsky and M. Litvinov, Metal or insulator? Dirac operator spectrum in holographic QCD, Phys. Lett. B 795 (2019) 379 [arXiv: 1812.02321] [InSPIRE].

[44] R.M. Nandkishore and M. Hermele, Fractons, Ann. Rev. Condensed Matter Phys. 10 (2019) 295 [arXiv:1803.11196] [INSPIRE].

[45] M. Pretko, The fracton gauge principle, Phys. Rev. B 98 (2018) 115134 [arXiv:1807.11479] [INSPIRE].

[46] H.-U. Yee and I. Zahed, Holographic two dimensional QCD and Chern-Simons term, JHEP 07 (2011) 033 [arXiv:1103.6286] [INSPIRE].

[47] D. Diakonov and M.I. Eides, Chiral Lagrangian from a functional integral over quarks, JETP Lett. 38 (1983) 433 [Pisma Zh. Eksp. Teor. Fiz. 38 (1983) 358] [InSPIRE].

[48] P. Rossi, Propagation functions in the field of a monopole, Nucl. Phys. B 149 (1979) 170 [INSPIRE].

[49] F. Wilczek, Quantum time crystals, Phys. Rev. Lett. 109 (2012) 160401 [arXiv:1202.2539] [INSPIRE].

[50] F. Wilczek, Superfluidity and space-time translation symmetry breaking, Phys. Rev. Lett. 111 (2013) 250402.

[51] K. Sacha and J. Zakrzewski, Time crystals: a review, Rept. Prog. Phys. 81 (2017) 016401.

[52] I.K. Affleck, O. Alvarez and N.S. Manton, Pair production at strong coupling in weak external fields, Nucl. Phys. B 197 (1982) 509 [InSPIRE].

[53] A. Gorsky, Spontaneous creation of the brane world and direction of the time arrow, Phys. Lett. B 646 (2007) 183 [hep-th/0606072] [InSPIRE].

[54] S. Kinoshita, K. Murata and T. Oka, Holographic Floquet states II: Floquet condensation of vector mesons in nonequilibrium phase diagram, JHEP 06 (2018) 096 [arXiv:1712.06786] [INSPIRE].

[55] N. Seiberg, Field theories with a vector global symmetry, arXiv:1909.10544 [INSPIRE]. 